\title{
Erratum: Upconversion Loop Oscillator Axion Detection Experiment: A Precision Frequency Interferometric Axion Dark Matter Search with a Cylindrical Microwave Cavity [Phys. Rev. Lett. 126, 081803 (2021)]
}

\author{
Catriona A. Thomson $\odot$, Ben T. McAllister, Maxim Goryachev, Eugene N. Ivanov, and Michael E. Tobar
}

(Received 10 May 2021; published 2 July 2021)

DOI: 10.1103/PhysRevLett.127.019901

We have found a sign error in our sensitivity calculation. Our results still experimentally demonstrate the feasibility of exploiting upconversion and downconversion to search for axions, but the sensitivity to low mass axions claimed in [1,2] and quoted in [3-5] must be substantially reduced by 4 orders of magnitude to $3 \times 10^{-3} 1 / \mathrm{GeV}$ between 7.44 and $19.38 \mathrm{neV}$ and by up to 11 orders of magnitude for the lowest axion masses in the theoretical projections in the left panel of Fig. 3 in the main text. Conversely, the sensitivity to high mass axions must be increased to $10^{-2} 1 / \mathrm{GeV}$ between 74.4 and $74.5 \mu \mathrm{eV}$. With this correction, our results show that calculating sensitivity using the photon-axion interaction Hamiltonian is consistent with using a derivative coupling to the axion, showing suppressed sensitivity as $m_{a} \rightarrow 0$. This is consistent with other work on axion searches in excited cavities [6,7]. Thus, our conclusion that measuring axion induced frequency shifts was much better than measuring axion induced power was in fact wrong.

The sign error occurs in Eq. (11) of the Supplemental Material, where the normalized unit vectors for the $\mathrm{TE}_{0,1,1}$ mode are given. Here, the negative sign before the $z$ component of $\boldsymbol{b}_{1}$ was missed. The correct expressions are

$$
\boldsymbol{e}_{1}=-\frac{\sqrt{2} J_{1}\left(\frac{\chi_{01}^{\prime}}{a} r\right) \sin \left(\frac{\pi z}{L}\right)}{J_{0}\left(\chi_{01}^{\prime}\right)} \hat{\varphi} \quad \text { and } \quad \boldsymbol{b}_{1}=\frac{\sqrt{2} \frac{a \pi}{L \chi_{01}^{\prime}} J_{1}\left(\frac{\chi_{01}^{\prime}}{a} r\right) \cos \left(\frac{\pi z}{L}\right)}{\sqrt{1+\left(\frac{a \pi}{L \chi_{01}^{\prime}}\right)^{2}} J_{0}\left(\chi_{01}^{\prime}\right)} \hat{r}-\frac{\sqrt{2} J_{0}\left(\frac{\chi_{01}^{\prime}}{a} r\right) \sin \left(\frac{\pi z}{L}\right)}{\sqrt{1+\left(\frac{a \pi}{L \chi_{01}^{\prime}}\right)^{2}} J_{0}\left(\chi_{01}^{\prime}\right)} \hat{z} .
$$

These expressions are used to derive the form factors $\xi_{ \pm}$and hence the conversion factors $k_{a \pm}$, which determine the sensitivities to downconverted (sum frequency) and upconverted (difference frequency) axions, respectively. The error therefore exchanges the values of $\xi_{ \pm}$, which are correctly written as

$$
\xi_{-}=\xi_{12}-\xi_{21}=\xi_{12}\left(1-\frac{f_{2}}{f_{1}}\right) \quad \text { and } \quad \xi_{+}=-\left(\xi_{12}+\xi_{21}\right)=-\xi_{12}\left(1+\frac{f_{2}}{f_{1}}\right)
$$

which is the corrected Eq. (14) in the Supplemental Material. Also, the error exchanges the values of $k_{a \pm}$,

$$
k_{a \pm}^{2}=\frac{32 \chi_{01}^{\prime 2}}{\left(\chi_{02}^{2}-\chi_{01}^{\prime 2}\right)^{2}} \frac{\beta_{1} P_{1} Q_{L 1}\left(\beta_{2}+1\right)^{2}}{\beta_{2} P_{2} Q_{L 2}\left(\beta_{1}+1\right)^{2}} \frac{\left(f_{2} \pm f_{1}\right)^{2}}{f_{1} f_{2}}
$$

which is the corrected Eq. (18) in the Supplemental Material. The quoted conversion factors in Table I of the main text should be reversed, with $k_{a+}=5.5$ and $k_{a-}=8.4 \times 10^{-4}-1.1 \times 10^{-3}$, and Fig. 3 in the Supplemental Material should be replaced by Fig. 1.

Hence, the quoted SNRs for the free running loop oscillator [Eq. (8) in the main text and Eq. (22) in the Supplemental Material) and the stabilized loop oscillator [Eq. (25) in the Supplemental Material] are written incorrectly. When the modes are closely tuned, the equations give the sensitivity to downconverted axions, with $f_{a_{-}}$replaced with $f_{a_{+}}$. Thus, given the condition that $f_{1}=f_{2}+\delta f_{12}$ is tuned slightly away from $f_{2}$ so that $\delta f_{12} / f_{2} \ll 1$, and assuming $\beta_{1}=1$, the corrected Eq. (8) in the main text [or Eq. (22) in the Supplemental Material] is

Published by the American Physical Society under the terms of the Creative Commons Attribution 4.0 International license. Further distribution of this work must maintain attribution to the author(s) and the published articles title, journal citation, and DOI. 

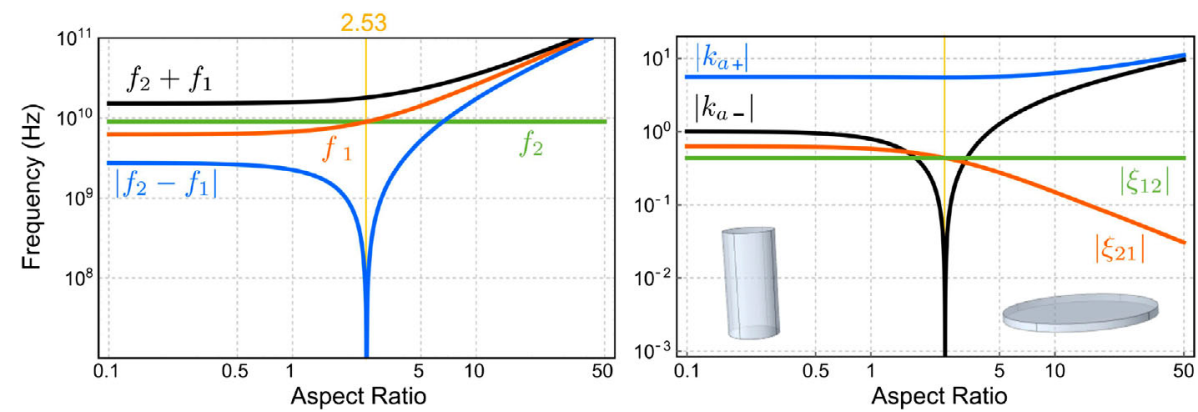

FIG. 1. Corrected Fig. 3 in the Supplemental Material of $k_{a \pm}$ conversion factors versus aspect ratio. Upconversion sensitivity is suppressed in the low axion mass case when the aspect ratio is 2.53 , as in the reported experiment.

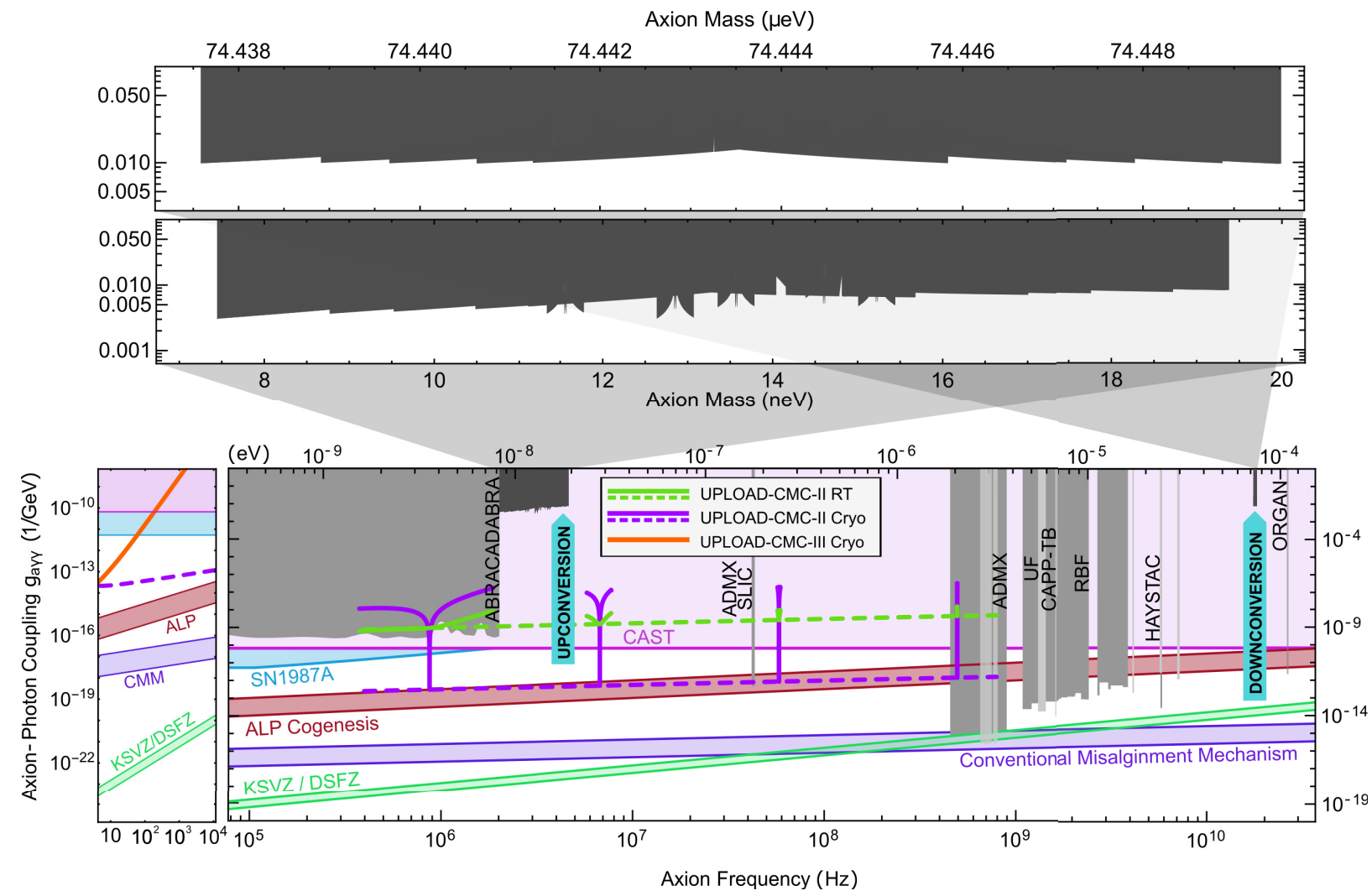

FIG. 2. Updated $95 \%$ confidence exclusion zones for $g_{a \gamma \gamma}$ for the measured upconversion and downconversion mass ranges (above), with CAST's helioscope limits in pink and QCD axion models in green (KSVZ and DSFZ) [8,9], purple (conventional ALP misalignment), and red (ALP cogenesis) [10,11]. Corrected upconversion limits are dashed, assuming $1 \mathrm{~Hz}$ tuning steps (30 days per $\mathrm{Hz}$ ): UPLOAD-CMC-II-RT, a copper resonator with frequency stabilized (FS) loops at room temperature (RT); and UPLOAD-CMC-IICryo, cryogenic $\mathrm{Nb}$ with FS loops. We also present examples of 30 day measurements covering $1 \mathrm{MHz}$ in Fourier space in bold. The left subplot displays (in orange) a 30 day measurement projection for UPLOAD-CMC-III-Cryo, which is simply the cryogenic FS setup when modes are tuned just $5 \mathrm{~Hz}$ apart. The results are compared to ADMX [12-15], ORGAN [16], ABRACADABRA [17], ADMXSLIC [18], HAYSTAC [19], UF [20], CAPP-8 TB [21], and RBF [22].

$$
\mathrm{SNR}_{-}=g_{a \gamma \gamma} \frac{2.7\left(\frac{10^{6} t}{f_{a_{-}}}\right)^{1 / 4} \sqrt{\rho_{\mathrm{DM}} c^{3}}}{2 \pi f_{a_{-}}} \sqrt{\frac{Q_{L 2} P_{\mathrm{amp}}\left(\beta_{2}+1\right)^{2}}{\left(F k_{B} T_{0}\right) \beta_{2} P_{2}}} \sqrt{P_{1} Q_{L 1}} \sqrt{\frac{1}{\left(2 Q_{L 2} \frac{f}{f_{2}}\right)^{2}+1}}\left(\frac{\left|\delta f_{12}\right|}{\sqrt{2} f_{2}}\right)
$$

which has an extra term at the end proportional to the detuning between the two modes. Likewise, Eq. (25) becomes 


$$
\mathrm{SNR}_{-}=g_{a \gamma \gamma} \frac{3.9\left(\frac{10^{6} t}{f_{a_{-}}}\right)^{1 / 4} \sqrt{\rho_{\mathrm{DM}} c^{3}}}{2 \pi f_{a_{-}}} \sqrt{\frac{Q_{L 1} P_{1}}{k_{b} T_{\mathrm{RS}}}} \sqrt{\frac{\beta_{2} Q_{L 2}}{\left(2 Q_{L 2} \frac{f}{f_{2}}\right)^{2}+1}}\left(\frac{\left|\delta f_{12}\right|}{\sqrt{2} f_{2}}\right) .
$$

Thus, we present the corrected exclusion plot for $g_{a \gamma \gamma}$ according to the corrected SNR equations in Fig. 2.

We are very grateful to Kevin Zhou for meticulously going through our paper and finding our mistake and for reading this correction to make sure of its validity. This work was funded by the ARC Centre of Excellence for Engineered Quantum Systems, CE170100009, and Dark Matter Particle Physics, CE200100008.

[1] C. A. Thomson, B. T. McAllister, M. Goryachev, E. N. Ivanov, and M. E. Tobar, UPconversion Loop Oscillator Axion Detection experiment: A precision frequency interferometric axion dark matter search with a Cylindrical Microwave Cavity, Phys. Rev. Lett. 126, 081803 (2021).

[2] M. Goryachev, B. T. McAllister, and M. E. Tobar, Axion detection with precision frequency metrology, Phys. Dark Universe 26, 100345 (2019).

[3] L. Darmé, L. Di Luzio, M. Giannotti, and E. Nardi, Selective enhancement of the QCD axion couplings, Phys. Rev. D 103, 015034 (2021).

[4] J. Billard, M. Boulay, S. Cebrián, L. Covi, G. Fiorillo, A. Green, J. Kopp, B. Majorovits, K. Palladino, F. Petricca et al., Direct detection of dark matter-Appec committee report, arXiv:2104.07634.

[5] L. J. Hall, K. Harigaya et al., Predictions for axion couplings from alp cogenesis, J. High Energy Phys. 01 (2021) 1.

[6] A. Berlin, R. Tito D’Agnolo, S. A. R. Ellis, C. Nantista, J. Neilson, P. Schuster, S. Tantawi, N. Toro, and K. Zhou, Axion dark matter detection by superconducting resonant frequency conversion, J. High Energy Phys. 07 (2020) 1.

[7] R. Lasenby, Parametrics of electromagnetic searches for axion dark matter, Phys. Rev. D 103, 075007 (2021).

[8] V. Anastassopoulos et al., New cast limit on the axion-photon interaction, Nat. Phys. 13, 584 (2017).

[9] P. W. Graham, I. G. Irastorza, S. K. Lamoreaux, A. Lindner, and K. A. van Bibber, Experimental searches for the axion and axionlike particles, Annu. Rev. Nucl. Part. Sci. 65, 485 (2015).

[10] R. T. Co, L. J. Hall, and K. Harigaya, Predictions for axion couplings from alp cogenesis, J. High Energy Phys. 01 (2021) 172.

[11] R. T. Co, L. J. Hall, and K. Harigaya, Axion Kinetic Misalignment Mechanism, Phys. Rev. Lett. 124, 251802 (2020).

[12] N. Du et al., Search for Invisible Axion Dark Matter with the Axion Dark Matter Experiment, Phys. Rev. Lett. 120, 151301 (2018).

[13] C. Boutan et al., Piezoelectrically Tuned Multimode Cavity Search for Axion Dark Matter, Phys. Rev. Lett. 121, 261302 (2018).

[14] T. Braine, R. Cervantes, N. Crisosto, N. Du, S. Kimes, L. J. Rosenberg, G. Rybka, J. Yang, D. Bowring, A. S. Chou, R. Khatiwada, A. Sonnenschein, W. Wester, G. Carosi, N. Woollett, L. D. Duffy, R. Bradley, C. Boutan, M. Jones, B. H. LaRoque et al., Extended Search for the Invisible Axion with the Axion Dark Matter Experiment, Phys. Rev. Lett. 124, 101303 (2020).

[15] C. Bartram, T. Braine, R. Cervantes, N. Crisosto, N. Du, G. Leum, L. J. Rosenberg, G. Rybka, J. Yang, D. Bowring, A. S. Chou, R. Khatiwada, A. Sonnenschein, W. Wester, G. Carosi, N. Woollett, L. D. Duffy, M. Goryachev, B. McAllister, M. E. Tobar et al. (ADMX Collaboration), Axion dark matter experiment: Run 1b analysis details, Phys. Rev. D 103, 032002 (2021).

[16] B. T. McAllister, G. Flower, E. N. Ivanov, M. Goryachev, J. Bourhill, and M. E. Tobar, The organ experiment: An axion haloscope above 15 GHz, Phys. Dark Universe 18, 67 (2017).

[17] J. L. Ouellet et al., First Results from Abracadabra-10 cm: A Search for Sub- $\mu$ ev Axion Dark Matter, Phys. Rev. Lett. 122, 121802 (2019).

[18] N. Crisosto, P. Sikivie, N. S. Sullivan, D. B. Tanner, J. Yang, and G. Rybka, Admx slic: Results from a Superconducting LC Circuit Investigating Cold Axions, Phys. Rev. Lett. 124, 241101 (2020).

[19] B. M. Brubaker et al., First Results from a Microwave Cavity Axion Search at $24 \mu \mathrm{ev}$, Phys. Rev. Lett. 118, 061302 (2017).

[20] C. Hagmann, P. Sikivie, N. S. Sullivan, and D. B. Tanner, Results from a search for cosmic axions, Phys. Rev. D 42, 1297 (1990).

[21] S. Lee, S. Ahn, J. Choi, B. R. Ko, and Y. K. Semertzidis, Axion Dark Matter Search Around $6.7 \mu \mathrm{eV}$, Phys. Rev. Lett. 124, 101802 (2020).

[22] W. U. Wuensch, S. De Panfilis-Wuensch, Y. K. Semertzidis, J. T. Rogers, A. C. Melissinos, H. J. Halama, B. E. Moskowitz, A. G. Prodell, W. B. Fowler, and F. A. Nezrick, Results of a laboratory search for cosmic axions and other weakly coupled light particles, Phys. Rev. D 40, 3153 (1989). 